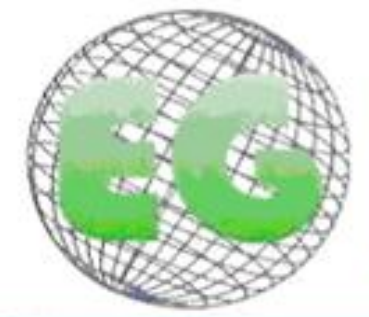

$N^{\circ} 39$

www.um.es/egloball

\title{
CLÍNICA
}

\section{Efectos de una intervención on line en conductas de autocuidado asociadas a la alimentación}

Effects of an intervention on line in self-care feeding behavior

\section{*Jaimovich, Sonia *Campos, Cecilia **Bustos, José ***Campos, María Sylvia ****Lillo, Verónica ${ }^{* * * * *}$ Herrera, Luz María ${ }^{* * * * *}$ Arellano, Valentina}

*Magister en Salud Pública, Profesora Titular Escuela de Enfermería Pontificia Universidad Católica de Chile. E-mail: sjaimovi@uc.cl **Biólogo Marino, Magister en Estadística Aplicada Universidad de Concepción. Director AESPRO (Análisis Estadístico Profesional). ${ }^{* * *}$ Magister en Ciencias de la Nutrición, Profesora Asociada Escuela de Enfermería Pontificia Universidad Católica de Chile.****Magister en Educación Especial, Profesora Asociada, Facultad de Educación, Pontificia Universidad Católica de Chile ${ }^{* * * * *}$ Magíster en Enfermería, Profesora Asistente Escuela Enfermería Pontificia Universidad Católica de Chile ${ }^{* * * * * *}$ Interna de Enfermería, Escuela de Enfermería Pontificia Universidad Católica de Chile, Santiago, Chile

Palabras Clave: Alimentación escolar; sobrepeso; obesidad; autocuidado; conducta alimentaria.. Keywords: School feeding; Overweight; Obesity; Self care; feeding behavior.

\section{RESUMEN}

Resultados del proyecto "Prevención del sobrepeso y de la obesidad en escolares chilenos: Apuesta a la capacidad emprendedora de los niños" (FONIS SA10I20016) en respuesta a la interrogante: ¿ES posible contribuir al cambio de conductas asociadas a sobrepeso y obesidad a través de una intervención diseñada para este fin?

Material y Métodos: Estudio cuasi experimental en niños/as de $5^{\circ}$ y $6^{0}$ año básico de colegios municipalizados, comuna La Pintana, Región Metropolitana, Chile. Previa obtención de consentimiento informado, se seleccionaron mediante muestreo aleatorio por conglomerado 6 colegios para grupo estudio $n=442$ y 6 colegios para grupo control $n=328$. La intervención, con apoyo de software en página Web, se realizó en función del auto diagnóstico de conductas asociadas a la alimentación, identificadas con el instrumento Moore Index Nutrition-Child (MIN-C()), diseñado de acuerdo al marco conceptual de la Teoría de Déficit de Autocuidado de Dorothea Orem. El análisis de resultados utilizó pruebas paramétricas con software R 2012.

Resultados: Existe efecto positivo del número de ingresos al sitio web sobre las conductas observadas en el post test comparadas con las del pretest en el grupo intervenido. 
Conclusión: El efecto de la intervención fue estadísticamente significativo para las conductas estudiadas, en los niños/as que utilizaron el software 8 y más veces. El marco teórico de Orem permite el diseño de una intervención dirigida a motivar el cambio de conductas asociadas a una alimentación saludable.

\section{ABSTRACT}

Results of the project "Prevention of overweight and obesity in Chilean school children: A bet on the enterprising capacity of children" (FONIS SA10I20016), in response to the question: Is it possible to help change behaviors associated with overweight and obesity through an intervention designed for this purpose?

Material and Methods: A quasi-experimental study in 5th and $6^{\text {th }}$ grade children of primary municipal schools, La Pintana, Metropolitan Region, Chile. After obtaining informed consent, 6 schools were selected by random cluster sampling for the study group $n=442$ and 6 schools for control group $n=$ 328. The intervention, with software supported in Web page, was performed according to the auto diagnostics of behaviors associated with feeding, identified with the Moore-Child Nutrition Index (MIN-C (C) instrument. The analysis of results used parametric tests with R 2012.

Results: There is a positive effect of number of times entered to the website on behaviors observed in the post test compared with the pretest in the intervention group.

Conclusion: The effect of the intervention was statistically significant for the studied behaviors, in children who used the software 8 times or more.

\section{INTRODUCCIÓN}

En Chile, al igual que en gran parte del mundo, la magnitud del sobrepeso y la obesidad, como problemas de Salud Pública, es preocupante. La OMS plantea que su prevalencia ha aumentado a un ritmo alarmante. La considera la epidemia del siglo y uno de los problemas de salud pública más graves del siglo XXI; que está afectando progresivamente a muchos países de bajos y medianos ingresos, sobre todo en el medio urbano. Cada vez es mayor la proporción de niños con sobrepeso que viven en países en desarrollo ${ }^{(1-5)}$. En nuestro país el problema se inicia lentamente hace más de un cuarto de siglo y la transición nutricional ha sido a gran velocidad en todas las edades ${ }^{(6)}$. Su actual prevalencia es superior a lo que se esperaba para $1^{\circ}$ básico en los objetivos sanitarios de Chile 2000-2010 y por lo contrario el problema se ha incrementado de $16 \%$ en el año 2000 a $19 \%$ en $2008{ }^{(7)}$. En el contexto de América Latina, Chile es uno de los países que presenta la mayor tasa de sobrepeso y obesidad y donde el incremento ha sido importante ${ }^{(8)}$

Las altas tasas de prevalencia de sobrepeso y obesidad a nivel mundial pueden ser interpretadas como un fracaso de las políticas sanitarias y de educación. Los estilos de vida sedentarios y una alimentación inadecuada en calidad y en cantidad son consideradas causas sociales de la obesidad, las que interactúan con las condiciones genéticas latentes. El Reporte Foresight 2007 plantea que existe evidencia y consenso de que la vida moderna se ha convertido en el principal factor desencadenante de la obesidad ${ }^{(9,10)}$. Investigaciones recientes demuestran que los entornos y las redes sociales contribuyen y afectan a la obesidad ${ }^{(11)}$. Como una de las sugerencias para revertir el problema del sobrepeso y la obesidad, Amigo plantea establecer estrategias nacionales de vida saludable desde la niñez, donde el cambio de dieta y la actividad física son acciones fundamentales ${ }^{(8)}$.

El presente trabajo comunica resultados del Proyecto Prevención del sobrepeso y de la obesidad en escolares chilenos: Una apuesta a la capacidad emprendedora de los 
niños Fonis SA10120016 ${ }^{(12)}$, diseñado para dar respuesta a la interrogante: ¿Es posible contribuir al cambio de conductas asociadas al sobrepeso y obesidad a través de una intervención diseñada para este efecto? En él, con el propósito de influir positivamente en la prevención de este problema, se estudió si una intervención con participación activa de estudiantes de $5^{\circ}$ y $6^{\circ}$ año de educación básica que involucra a profesores, contribuyó al cambio de conductas asociadas a una alimentación saludable.

La intervención, como recurso preventivo, contempla el uso de un software embebido en una página Web. Por medio de éste, los niños autoevalúan su alimentación, planifican y ajustan su dieta de acuerdo a las recomendaciones, visualizan gráficamente cuán cerca de las recomendaciones de macronutrientes se encuentra su dieta, de modo que ellos mismos puedan corregirla si es necesario, y diseñan planes de cambio de conducta a través de formulación de compromisos. La intervención se realiza en función del auto diagnóstico de conductas de autocuidado asociadas a la alimentación, utilizando el instrumento Moore Index Nutrition-Child (MIN-C@) diseñado por JB Moore, a partir del marco conceptual de la Teoría de Déficit de Autocuidado de Dorothea Orem; que ha sido aplicado en escolares de USA, Nicaragua y Chile ${ }^{(13-15)}$

Se trabajó durante 15 semanas en las 12 escuelas municipalizadas de comuna de La Pintana, con los estudiantes de 6 de ellas como grupo en estudio y 6 como grupo control. Esta es una de las comunas de la Región Metropolitana de Chile, ubicada en la zona sur de Santiago; se trata de una comuna que se encuentra considerada de alta vulnerabilidad social. En esta intervención, el rol que deben desempeñar los profesores como referentes y líderes en el fortalecimiento y consolidación de conductas saludables es fundamental para su éxito.

\section{MATERIAL Y MÉTODO}

Diseño cuasi experimental prospectivo, con grupo control no equivalente, aleatorizado por conglomerados, que consideró a las escuelas como unidad de aleatorización. En las 12 escuelas municipalizadas de la comuna de La Pintana, previa obtención de consentimiento informado por parte de los niños y sus apoderados, los estudiantes de 6 de ellas (grupo en estudio), con un total de 442 niños/as, se sometieron a una intervención por 3-4 meses y se compararon con los de 6 escuelas de similares características (grupo control) con un total de 328 niños/as. Previo a la intervención como recurso preventivo y con el apoyo de un software embebido en una página Web denominado MeKuido, se realizaron las mediciones basales de estado nutricional y conductas de autocuidado. La medición del estado nutricional se realizó según las Normas de Evaluación Nutricional del Niño de 6 a 18 años propuestas por el Ministerio de Salud chileno el año $2003^{(16)}$. Esta normativa propone que los indicadores para la evaluación sean: Índice de Masa Corporal para la edad (IMC/E) y Talla para la Edad (T/E); ajustado el valor del IMC en función del grado de desarrollo puberal o los estadios de Tanner. La medición de las conductas asociadas a la alimentación se realizó con el instrumento MIN-C@, validado en español ${ }^{(14,17)}$, que se aplicó nuevamente post intervención para medir su efecto. En la aplicación post intervención respondieron el instrumento 379 niños/as del grupo estudio y 300 del grupo control. La disminución de niños/as se explica por inasistencias y retiro del colegio durante el periodo del estudio. Este instrumento fue diseñado de acuerdo al marco conceptual de la Teoría de Déficit de Autocuidado de Dorothea Orem ${ }^{(13,14)}$ y contiene 3 subescalas: una de operaciones estimativas, que mide preferentemente el interés por obtener información sobre el tema; otra de operaciones transicionales, que 
mide preferentemente la intención de realizar una determinada acción; y una tercera de operaciones productivas, que mide las acciones que ya se están ejecutando. Los estudiantes, supervisados por un profesor, trabajaron durante 15 semanas con el software MeKuido, alternándolo con sesiones grupales con el profesor/a.

Para medir el efecto de la intervención, se realizó una comparación de las conductas estudiadas a nivel intragrupo e intergrupo. Para comparar el puntaje promedio obtenido en la escala total y en cada una de sus subescalas en ambos grupos (control vs. intervenido) y antes y después de la intervención, se utilizaron análisis de la varianza de una vía de medidas repetidas en el tiempo, previa verificación de supuestos del análisis, mediante pruebas de normalidad de Shapiro-Wilks y pruebas de homogeneidad de varianzas de Levene ${ }^{(18)}$. Debido a que en la comparación de la Escala Total y de las subescalas se observaron diferencias marginales intragrupo, se buscó identificar el efecto del número de veces que los niños/as utilizaron el sitio web. Por lo tanto, en el grupo estudio se categorizaron dos nuevos grupos de niños/as: quienes ingresaron menos de 8 veces al sitio web (ingresos insuficientes) y quienes ingresaron 8 o más veces (ingresos suficientes). Se eliminaron del análisis los niños que no ingresaron. De esta forma, el nuevo grupo quedó conformado por 289 niños/as: 195 del grupo ingresos insuficientes y 94 del grupo ingresos suficientes. La comparación entre ambos grupos, para la escala total y las subescalas, medidas antes y después de la intervención, se realizó mediante análisis de la varianza factorial de 2x2, previa verificación de supuestos del análisis, mediante pruebas de normalidad de Shapiro-Wilks y pruebas de homogeneidad de varianzas de Levene ${ }^{(18)}$. Todas las pruebas estadísticas fueron realizadas considerando un valor alfa del $5 \%$ mediante el software estadístico $\mathrm{R}, 2012^{(19)}$.

Las conductas, como variable ordinal, se categorizaron como conductas no saludables si la puntuación fue de 1 o 2 en escala de Likert, y saludables cuando fue de 4 o 5. Para la comparación del promedio obtenido por pregunta antes y después de la intervención se utilizó la prueba t de Student.

El proyecto Prevención del sobrepeso y de la obesidad en escolares chilenos: Una apuesta a la capacidad emprendedora de los niños Fonis SA10I20016, fue aprobado por el Comité de Ética de la Escuela de Enfermería de la Pontificia Universidad Católica de Chile.

\section{Instrumento:}

La variable dependiente prácticas de autocuidado asociadas a la alimentación se estudió con el Moore Index of Nutrition-Child (MIN-C) ${ }^{(20)}$; disponible en inglés y español. Está compuesto por 50 items en escala de Likert con 5 alternativas de respuesta, que miden frecuencia de comportamientos. Los puntajes más altos indican prácticas asociadas a la alimentación más saludable. La confiabilidad de la versión en español, adaptada en Chile por autoras de este proyecto, tuvo un coeficiente alfa de $.89^{(21)}$. En este artículo se presentan resultados de los primeros 42 items, considerada para su análisis como "escala total", con un puntaje ideal de 210 puntos, de ellos 13 items corresponden a operaciones estimativas, 12 a transicionales y 17 a productivas que conforman las 3 subescalas. Las alternativas de respuesta son nunca, rara vez, algunas veces, casi siempre y siempre. No se incluyen resultados referidos a 8 items de la escala que miden frecuencia de consumo de determinados alimentos, cuya categoría de respuesta es diferente (nunca, una o dos veces por semana, una vez al día, dos veces al día y 3 o más veces al día). 


\section{RESULTADOS}

\section{Descripción del grupo estudiado}

El diagnóstico basal se realizó con 796 niños/as. De ellos, un 52\% corresponde a mujeres y un $48 \%$ a hombres. Sus edades fluctuaron entre 9 y 15 años, con un promedio de 11. La evaluación del estado nutricional mostró que un $43 \%$ de los niños/as se encontraba en parámetros normales, un $36 \%$ en riesgo de obesidad con valores similares en ambos sexos, y un 19\% con obesidad. Se encontró mayor proporción de mujeres que hombres con obesidad, $21 \%$ vs $17 \%$. Solo un $2 \%$ de los niños/as se encontró con bajo peso. Respecto a las prácticas de autocuidado, la medición basal mostró que en la mayoría de los casos estas eran regulares $(90.6 \%)$. Solo 20 niños/as presentaban prácticas saludables $(2.5 \%)$ y 55 presentaban prácticas no saludables (6.9\%). El puntaje promedio obtenido en los primeros 42 items de la escala del MIN-C@ fue de 132 puntos, de un puntaje ideal de 210, que representa un $62.9 \%$.

\section{Comparación grupo intervenido y grupo control}

El análisis de la varianza de medidas repetidas tanto para los 42 items estudiados (escala total) del MIN-C $\odot$ como de los ítems de las 3 sub escalas que la conforman, muestra que si bien existe una tendencia a aumentar el puntaje en el post test, la diferencia entre ambos grupos (control vs intervenido) no es significativa pues el valor de probabilidad del factor grupos es superior al 0,05.

\section{Comparación intra grupo intervenido}

En el grupo intervenido es posible identificar una relación positiva entre el número de ingresos al sitio Web y la diferencia del puntaje promedio entre el pre y post test, pero no lo suficientemente fuerte para alcanzar una significancia estadística. Por esta razón, se dividió a los niños/as en dos categorías: Insuficiente y Suficiente.

De acuerdo a esta nueva clasificación del grupo intervenido, se identificaron 205 niños/as en la categoría insuficiente y 94 niños en la categoría suficiente. Al comparar ambos grupos, se observaron diferencias significativas en la escala total, y en cada una de las subescalas.

Para la escala total (42 ítems), la tasa de cambio es mayor para el grupo de niños/as catalogado como Suficiente en comparación con el grupo catalogado Insuficiente, diferencia estadísticamente significativa $F(1,574)=7.62 ; p<0.05$. No se evidenció efecto del tiempo por sí solo entre el pre y el post test. (Gráfico 1) 


\section{Gráfico 1}

Comparación pre y post test entre grupos con ingresos Suficientes vs Insuficientes Escala Total (42 items)

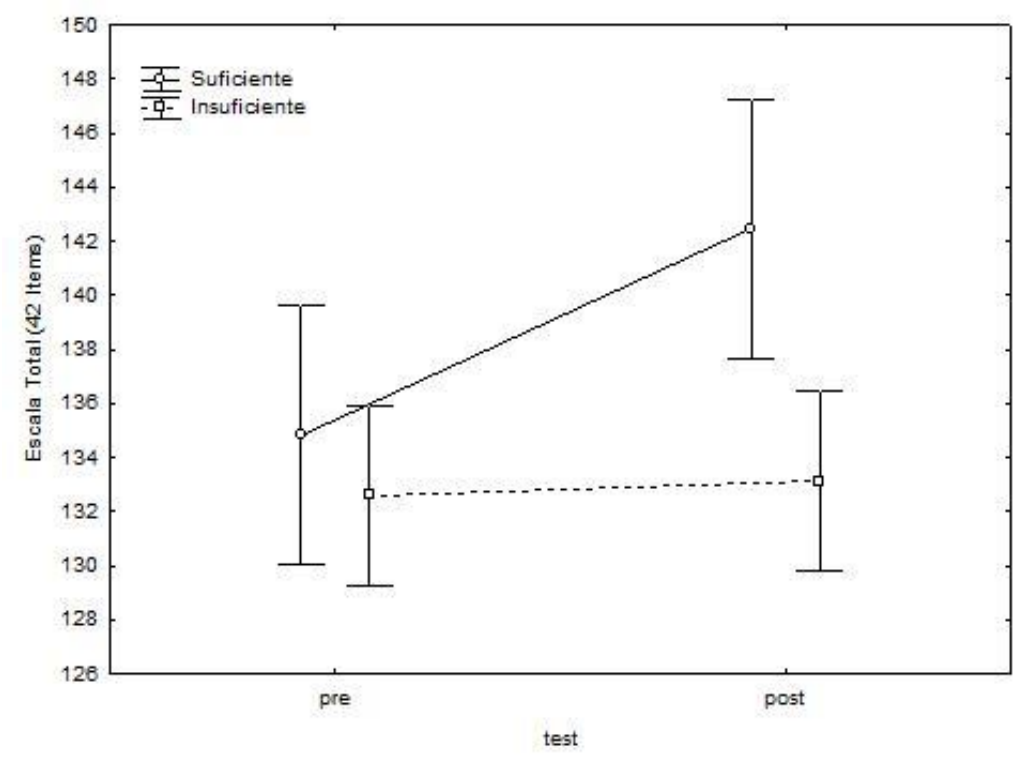

Las barras indican un intervalo de confianza del 95\%

Para la escala de operaciones estimativas (13 items), el tiempo afectó más fuertemente al grupo Suficiente, que a su vez difiere estadísticamente del grupo Insuficiente. Se observan diferencias significativas en la interacción entre el tiempo por sí solo y el grupo $(F(1,574)=4.29 ; p<0.05)$, para el nivel de ingreso (Suficiente vs Insuficiente) $F(1,574)=5.18 ; p<0.05$, como también en el efecto del tiempo entre el pre y el post test $F(1,574)=8.62 ; p<0.05$. (Gráfico 2) Entre los ítems de esta subescala que mostraron un cambio significativo entre el pre y post test se puede mencionar "le hablo a mis amigos acerca de los alimentos saludables que debemos consumir" (t-value $-2.044 p<0.05)$.

\section{Gráfico 2}

Comparación pre y post test entre grupos con ingresos Suficientes vs Insuficientes Subescala Operaciones Estimativas (13 items)

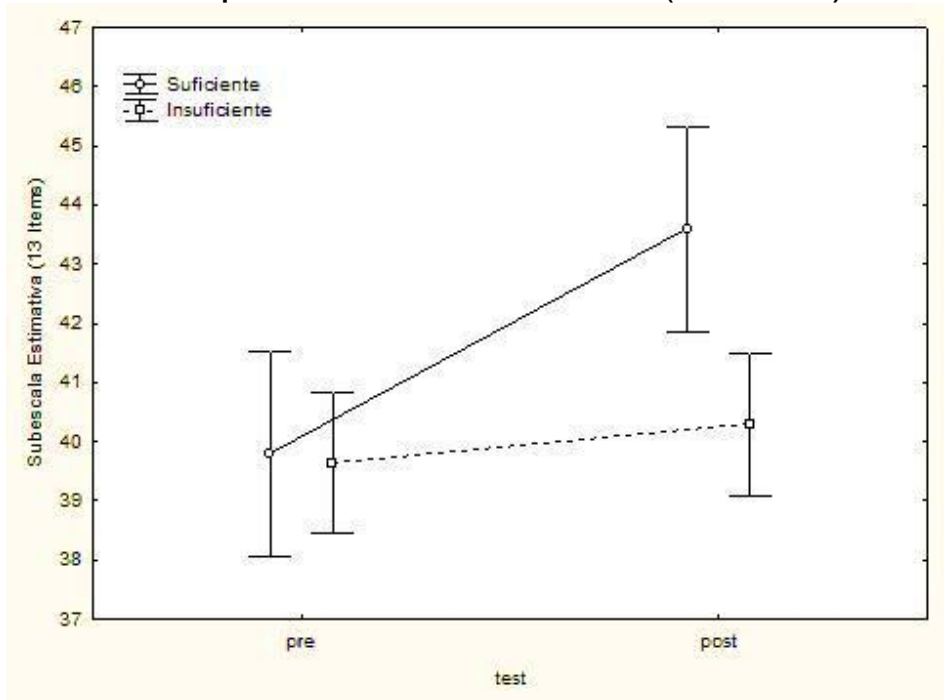

Las barras indican un intervalo de confianza del 95\% 
Para la escala de operaciones transicionales (12 items) se observa una tendencia al aumento en ambos grupos (Suficiente vs Insuficiente), pero es mayor en el grupo Suficiente y las diferencias entre los grupos son significativas $(F 1.574=1.72 ; p<$ 0.05). (Gráfico 3). Entre los ítems de esta subescala que mostraron un cambio significativo entre el pre y post test se pueden mencionar el aumento de puntaje en "elijo comer alimentos que contienen vitaminas" (t-value $-2.375 p<0.05$ ) y "yo evalúo si mis comidas tienen suficiente proteína" (t-value $-2.894 \mathrm{p}<0.05)$ y la disminución del puntaje en "tomo gaseosa en vez de refrescos naturales" (t-value $-1.789 \mathrm{p}<0.05$ ), "prefiero comer dulces en vez de frutas" ( $\mathrm{t}$-value $-2.345 \mathrm{p}<0.05)$ y "prefiero comer golosinas en vez de frutas" (t-value $-2.184 \mathrm{p}<0.05)$.

\section{Gráfico 3}

Comparación pre y post test entre grupos con ingresos Suficientes vs Insuficientes Subescala Operaciones Transicionales (12 items)

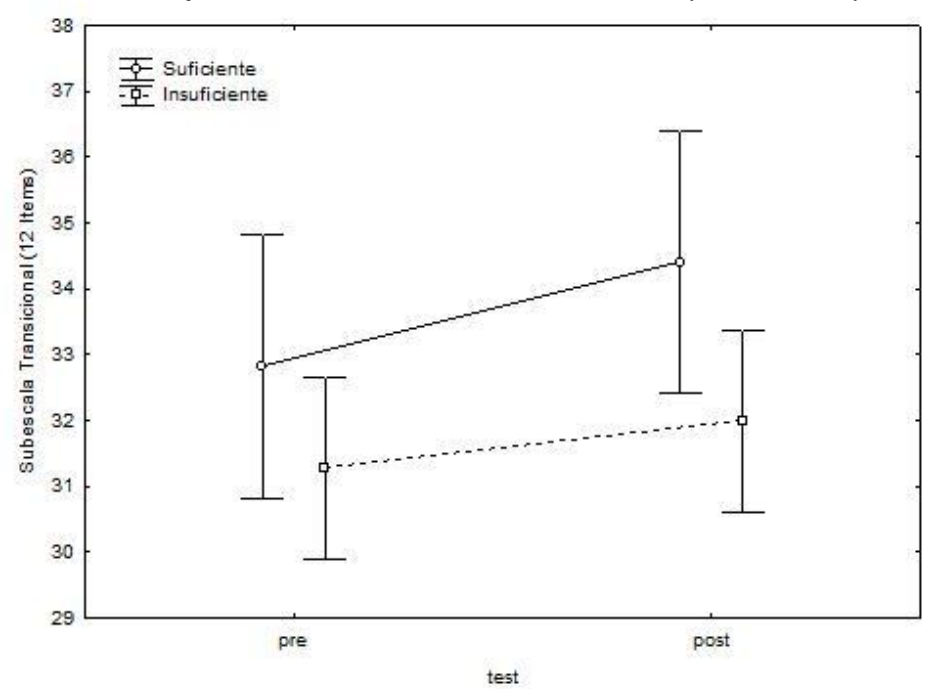

Las barras indican un intervalo de confianza del 95\%

Para la escala de operaciones productivas (17 ítems) se observan diferencias estadísticamente significativas entre ambos grupos $(F(1,574)=669 ; p<0.05)$, mientras que no se evidenció efecto del tiempo por sí solo. (Gráfico 4) Entre los ítems de esta subescala que mostraron un cambio significativo entre el pre y post test se pueden mencionar "consumo alimentos que contienen hierro" ( $t$-value $-2.375 \quad p<0.05)$ y "consumo alimentos que contienen proteína en cada comida" (t-value -2.044 $p<0.05$ ). 


\section{Gráfico 4}

Comparación pre y post test entre grupos con ingresos Suficientes vs Insuficientes Subescala Operaciones productivas (17 items)

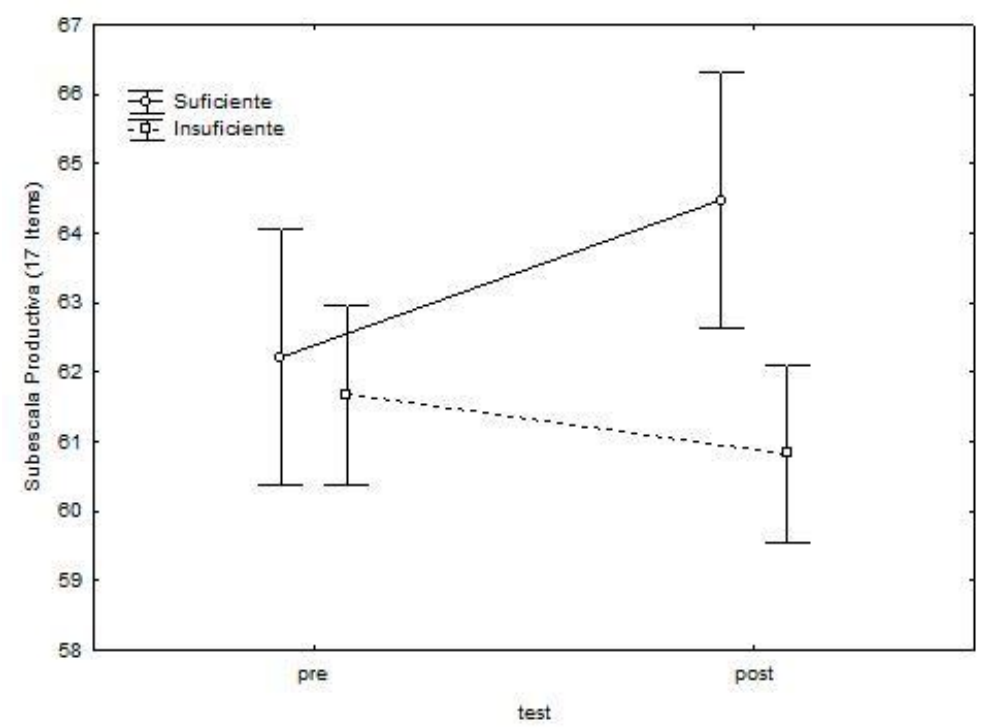

Las barras indican un intervalo de confianza del 95\%

\section{DISCUSIÓN}

Las conductas de alimentación se han deteriorado en los últimos años y se requiere la búsqueda de estrategias para revertir esto. Se han realizado variadas intervenciones en colegios para modificar los hábitos alimentarios y promover la actividad física con el fin de reducir la prevalencia de obesidad. La mayoría de ellas se centra en educación sobre el tema y fomento de la actividad física y consisten principalmente en estudios observacionales, con un corto periodo de observación, por lo que no se alcanza a ver resultados a nivel del Índice de Masa Corporal ${ }^{(22)}$. El cambio de conducta es un componente fundamental para lograr cualquier respuesta frente al sobrepeso y a la obesidad. Sin embargo, es un proceso complejo que va más allá de la educación y entrega de información. Se hacen necesarios cambios en el ambiente para prevenir conductas poco saludables. La evidencia sugiere que las estrategias actualmente utilizadas están fallando en la obtención de impacto suficiente porque no cubren todas las variables que influyen ${ }^{(9)}$. La mayoría de los tratamientos de obesidad han resultado poco efectivos y no perduran en el tiempo ${ }^{(23)}$. Por ello, dada la dificultad para lograr cambios de conducta en esta área, el énfasis para abordar este problema debe situarse en la promoción de estilos de vida saludable y en la prevención. La innovación en este proyecto está basada en los modelos de aprendizaje integral que contemplan los factores contextuales, cognitivos y afectivos; la participación y la incorporación de medios de comunicación en la intervención.

Este estudio aporta información relativa a la prevención del sobrepeso y obesidad en escolares chilenos, centrándose en las prácticas o conductas de autocuidado relacionadas con la alimentación y la capacidad de los niños/as para modificarlas, más que en la entrega de contenidos educativos y la práctica de actividad física. El diagnóstico basal realizado a través del MIN-C() mostró que los niños presentan prácticas que requieren ser mejoradas. El resultado es similar al encontrado en un estudio previo realizado en nuestro país para validar el instrumento ${ }^{(17)}$ y al encontrado 
por su autora en Washington DC ${ }^{(21)}$. Esto muestra la importancia de continuar en la búsqueda de las mejores estrategias que permitan modificar esta situación.

Entre las intervenciones que se han realizado con éxito para este fin se pueden citar: Un estudio de 2 años de duración que realizó una intervención educacional en hábitos alimenticios saludables y actividad física como actividad extracurricular, en escolares de $5^{\circ}$ y $6^{\circ}$ básico de España, encontró una disminución del aumento del IMC y cambios favorables en hábitos alimenticios en niños del grupo intervenido en comparación con el grupo control (24). Fahlman, Dake, McCaughtry y Martin encontraron que el grupo de estudiantes con promedio de edad de 12 años, intervenido con un Modelo de Curriculum de Nutrición en Michigan, mejoraba sus conocimientos post intervención y que posteriormente tenía mayor disposición hacia el consumo de frutas y verduras y menor disposición hacia la comida chatarra que el grupo control, así como mayor confianza en consumir alimentación saludable ${ }^{(25)}$. Un estudio piloto realizado en Washington por la autora del instrumento MIN-C(C), utilizado en el presente trabajo, estudió el efecto de un programa de educación en nutrición denominado Color My Pyramid, dirigido a niños/as de $4^{\circ}$ y $5^{\circ}$ año básico. Analizaron conocimientos, prácticas de autocuidado, niveles de actividad física y estado nutricional en un estudio cuasi experimental. La intervención utilizó el componente on line www.MyPyramid.gov y la teoría de Déficit de Autocuidado de D. Orem. Los resultados mostraron un incremento en los conocimientos de nutrición y tiempo de actividad física en el periodo entre pre y postest ${ }^{(20)}$. En Chile, se aplicó el Programa Desafío Chileno para una Vida Activa en escolares de Casablanca, consistente en educación alimentario-nutricional aplicando metodologías y materiales educativos específicos y actividad física en el quehacer habitual. Si bien los cambios de peso fueron significativos en los primeros 2 años de seguimiento, la intervención no fue sustentable en el tiempo ${ }^{26,3)}$. Rajalakshmi, Lakshman, Sharp, Ong y Forouhi desarrollaron un juego de cartas para enseñar nutrición a niños de escuelas primarias en Gran Bretaña y demostraron que este juego facilita el aprendizaje. También encontraron mejores hábitos en el grupo intervenido que en el grupo control al finalizar la intervención de 9 semanas ${ }^{(27)}$

Estas diferentes estrategias que apuntan a la prevención del sobrepeso en escolares son una muestra de que si se incorporan como actividades regulares en los establecimientos educacionales, se obtienen resultados positivos. La estrategia MeKuido es una más de ellas, pero demuestra que si se usa en forma sistemática, de una forma entretenida y poco sofisticada, los niños/as se interesan en el tema y realizan modificación en sus prácticas de autocuidado. El desafío está en buscar la forma en que se incorpore la estrategia en los planes educacionales de los colegios.

El estudio presentó algunas limitantes importantes como fueron:

Retiro de un colegio a partir de 2011, con lo que se contó con 12 establecimientos en lugar de 13 como estaba programado; la no aceptación de participar en el estudio de un número no despreciable de niños, junto con la pérdida o falta de devolución de Consentimiento Informado por parte de niños y apoderados, lo que disminuyó el tamaño muestral con el que se pensaba trabajar, sin embargo la potencia de los resultados no se vio afectada; la falta de cumplimiento de una adecuada programación de profesores y directivos que limitó el número de veces en que los niños asistieron a la sala de computación junto a sus profesores, disminuyendo a 94 el número de niños que realmente utilizó el software 8 y más veces, en los que sí se 
observó un cambio significativo hacia prácticas favorables relacionadas a la alimentación.

Además de las limitantes, se identifican como fortalezas del estudio, por una parte que el que el instrumento utilizado para medir las prácticas de autocuidado en los niños fue de fácil comprensión, y que puede ser utilizado para medir el efecto de intervenciones dirigidas a prevenir el sobrepeso y la obesidad en nuestro país, y por otra que se utilizó un software de diseño simple y atractivo en colegios de sectores vulnerables de la Región Metropolitana de Chile, con el que los niños/as pueden trabajar sin dificultad.

\section{CONCLUSIONES}

Es posible contribuir al cambio de conductas asociadas al sobrepeso y obesidad en estudiantes de $5^{\circ}$ y $6^{\circ}$ año de educación básica de la comuna de La Pintana, Región Metropolitana de Chile, a través de una intervención con participación activa de estudiantes y que involucra a profesores, si el software diseñado para este fin es utilizado en forma sistemática, trabajando en él al menos 8 veces.

El marco teórico de Orem, con el que está construido el instrumento MIN-CC permite el diseño de una intervención dirigida a motivar al cambio de conductas asociadas a una alimentación saludable.

\section{Agradecimientos}

Los autores desean expresar su agradecimiento a: La llustre Municipalidad de La Pintana, a los directores de los colegios, sus profesores, alumnos participantes y sus apoderados; los estudiantes de la Escuela de Enfermería y de la Facultad de Educación que colaboraron en calidad de ayudantes; Marcela Urrutia y Gabriela Bucaray, tesistas del proyecto por la información aportada; Dayann Martínez y María Paz del Río por su participación en el proceso de recolección de la información y la Comisión Nacional de Investigación Científica y Tecnológica y su Fondo Nacional de Investigación y Desarrollo en Salud y la Pontificia Universidad Católica de Chile quienes hicieron posible la realización de este estudio.

\section{REFERENCIAS}

1 Gobierno de Chile Ministerio de Salud. Estrategia Global Contra la Obesidad / EGO [internet]. Chile. Disponible en: http://webhosting.redsalud.gov.cl/minsal/archivos/alimentosynutricion/ego/EGO CHILE.p $\mathrm{df} ? \mathrm{cbc}=56,57,202 \& \mathrm{id}=1659$

2 Albala C, Vio F. Epidemiología de la obesidad en Chile. Rev.chil.nutr. 2000; 27(1), 97-103

3 Vio F, Salinas J. Promoción de Salud y Calidad de Vida en Chile: Una política con nuevos desafíos. Rev. chil. nutr. [internet]. 2006 Disponible en: http://www.scielo.cl/scielo.php?script=sci_arttext\&pid=S0717-

75182006000300006\&lng=es. doi: 10.4067/S0717-75182006000300006.

4 Flegal KM. The obesity epidemic in children and adults: current evidence and research issues. Med Sci Sports Exerc. 1999 Nov; 31(11):509-14.

5 Organización Mundial de la Salud. Estrategia mundial sobre régimen alimentario, actividad física y salud. Sobrepeso y obesidad infantiles. 2012 disponible en http://www.who.int/dietphysicalactivity/childhood/es/index.html 
6 Mardones F. Obesidad en la niñez en Chile: un tema pendiente. Centro de Políticas Públicas UC. 2010 (5) 41.

7 Ministerio de Salud. Obesidad en Chile, Un Problema de Salud Pública Chile; 2008.

8 Amigo $\mathrm{H}$. Obesidad en el niño en América Latina: situación, criterios de diagnóstico y desafíos. Cad. Saúde Pública, 2003;19(Sup. 1):163-70.

9 Foresight. Tackling Obesities: Future Choices - Project report. United Kingdom: Department of Innovation Universities and Skills. 2007; available form: http://www.bis.gov.uk/assets/foresight/docs/obesity/17.pdf

10 Christakis N, Fowler J. The Spread of Obesity in a Large Social Network over 32 Years. N Engl J Med. 2007 357:370-379 doi: 10.1056/NEJMsa066082

11 Parysow R. «Aspectos psicológicos en Obesidad». Un intento de interpretación. [tesina]. Departamento de Investigación Universidad de Belgrano;2005 Recuperado de http://www.ub.edu.ar/investigaciones/tesinas/172 parysow.pdf

12 Conicyt. Proyecto FONIS 10120016 Prevención del sobrepeso y la obesidad en escolares chilenos: una apuesta a la capacidad emprendedora de los niños. 2010

13 Orem D. Nursing: concepts of practice. 6 ed. Saint Louis: Mosby; 2001. pp. 7-9, 226, 541

14 Moore JB, Pawloski L, Baghi H., Whitt K, Rodriguez C, Lumbi, Bashatah A. Development and examination of psychometric properties of self-care instruments to measure nutrition practices for English and Spanish-speaking adolescents: Self Care Depend Care Nurs.2005, 13(1), 9-16.

15 Campos C, Jaimovich S, Moore J, Pawloski L, Gaffney K, González M. Operaciones de Autocuidado asociadas a la nutrición en madres de escolares chilenos. Horizonte Enferm -Chi-. 2008; 19(2):45-53.

16 Gobierno de Chile, Ministerio de Salud. Norma técnica de evaluación nutricional del niño de 6 a 18 años, 2003. Disponible en http://www.redsalud.gov.cl/archivos/alimentosynutricion/estrategiaintervencion/Norma EvNut6a18anos.pdf

17 Jaimovich S, Campos C, Campos MS, Moore J, Pawloski L, Gaffney K. Estado Nutricional y Conductas Asociadas a la Nutrición en Escolares. Rev Chil Pediatr. 2010; 81 (6): 515-522

18 Sokal R, Rohlf J. Biometry: the principles and practice of statistics in biological research. $3^{\text {th }}$ ed., New York: Freeman and Company New York, USA. 1995,

19 Wirtschafts Universitat wien Vienna University of economics and business. Institute for Statistics and mathematics. The R Project for Statistical Computing. Disponible en http://www.R-project.org/

20 Moore JB, Pawloski L, Goldberg P, Kyeung M, Stoehr A, Baghi H. Childhood Obesity Study: A Pilot Study of the Effect of the Nutrition Education Program Color My Pyramid. J Nurs. Sch. 2009 jun 25; 3:230-239.

21 Moore JB, Gaffney K, Pawloski L, Jaimovich S, Campos C. Maternal-Child Nutrition Practices and Pediatric Overweight/Obesity in the United States and Chile: A Comparative Study. J Pediatr Nursing. 2011 Oct 27 (5) 44-49

22 Kropski JA, Keckley PH, Jensen GL. School-based obesity prevention programs: an evidence-based review. Obesity (Silver Spring). 2008 May;16(5):1009-18. Epub 2008 Feb 28.

23 Llargues E, Franco R, Recasens A, Nadal A, Vila M, Perez MJ, Recasens I, et al. Assessment of a school-based intervention in eating habits and physical activity in school children: the AVall study J Epidemiol Community Health 2011;65:896e901. doi:10.1136/jech.2009.102319 
24 Moraga, F., Rebollo, MJ., Bórquez, P., Cáceres, J., Castillo, C.. Tratamiento de la obesidad infantil: Factores pronósticos asociados a una respuesta favorable Rev Chil Pediatr. 2003: 74 (4); 374-380.

25 Fahlman MM, Dake JA, McCaughtry N, Martin J. A pilot study to examine the effects of a nutrition intervention on nutrition knowledge, behaviors, and efficacy expectations in middle school children. J Sch Health. 2008; 78: 216-222.

26 Kain J, Uauy R, Leyton D, Cerda R, Olivares, S. y Vio F. Efectividad de una intervención en educación alimentaria y actividad física para prevenir obesidad en escolares de la ciudad de Casablanca, Chile (2003-2004) Rev. méd. Chile [revista en la Internet]; 2008 136(1): 22-30. Disponible en: http://www.scielo.cl/scielo.php?script=sci_arttext\&pid=S003498872008000100003\&lng=es. doi: 10.4067/S0034-98872008000100003

27 Rajalakshmi R, Lakshman S, Sharp J, Ong K, Forouhi N. A novel school-based intervention to improve nutrition knowledge in children: cluster randomised controlled trial BMC Public Health 2010, 10:123

Recibido: 16 de septiembre 2014; Aceptado: 10 octubre 2014

ISSN 1695-6141

(C) COPYRIGHT Servicio de Publicaciones - Universidad de Murcia 